\title{
Comparación de la atención del parto normal en los sistemas hospitalario y tradicional
}

Rosa María Méndez-González, MISS, ${ }^{(1)}$ María Dolores Cervera-Montejano, PhD. ${ }^{(1)}$

\section{Méndez-González RM, Cervera-Montejano MD. Comparación de la atención del parto normal en los sistemas hospitalario y tradicional. Salud Publica Mex 2002:44:129-136.} El texto completo en inglés de este artículo está disponible en: http://www.insp.mx/salud/index.html

\begin{abstract}
Resumen
Objetivo. Comparar la atención del parto por vía vaginal entre los sistemas hospitalario y tradicional, para identificar recursos y procedimientos utilizados, y la aparición de complicaciones mater nas y neonatales derivadas del tipo de atención. Material y métodos Estudio transversal realizado en tres hospitales de la ciudad de Mérida y cuatro municipios del estado de Yucatán, México, entre 1989 y 1990. La muestra estuvo constituida por 205 mujeres que tuvieron parto por vía vaginal. Se observó la atención del parto y, a los 15 días posparto, se les entrevistó para detectar complicaciones maternas y neonatales. Se calcularon proporciones y se aplicó ji cuadrada para compararlas. Resultados Se presentaron complicaciones en ambos sistemas. Sin embargo, en el hospitalario predominaron las maternas y en el tradicional, las neonatales. El número total de complicaciones fue similar. Conclusiones. La calidad de la atención fue similar en ambos servicios. Las complicaciones observadas pueden atribuirse a los recursos y acciones utilizados en cada sistema. Se requieren más estudios de este tipo para contar con evaluaciones objetivas de las ventajas y desventajas de ambos sistemas y contribuir a mejorar la calidad de la atención materno-infantil. El texto completo en inglés de este artículo está disponible en: http://www.insp.mx/salud/ index.html
\end{abstract}

Palabras clave: parto domiciliario/complicaciones; parto natural/complicaciones; atención hospitalaria/complicaciones; parteras tradicionales; México

\author{
Méndez-González RM, Cervera-Montejano MD. \\ A comparison of vaginal delivery care \\ between hospital and traditional systems. \\ Salud Publica Mex 2002;44:129-136. \\ The English version of this paper \\ is available at: http://www.insp.mx/salud/index.html
}

\begin{abstract}
A bst ract
Objective.To compare vaginal delivery hospital and traditional care systems to identify resources and practices, as well as maternal and neo natal complications related to each system. Material and Methods Between 1989 and 1990, a cross-sectional study was conducted in three hospitals of Merida City and four municipalities of the state of Yucatan. The study sample consisted of 205 women who had a normal vaginal delivery. Delivery procedures were observed and a questionnaire to identify complications was applied 15 days after childbirth. Data analysis consisted in comparison of proportions with the chi-squared test. Results Maternal and neo natal complications were identified in both systems; however, maternal complications were more frequent in hospital care, whereas neo natal complications were more frequent in traditional delivery care.The total number of complications was similar in the two systems. Conclusions. $N$ o difference was found in the quality of care between the hospital and the traditional systems. 0 bserved complications may be attributable to resources and practices of each system. Additional studies should be conducted to evaluate the advantages and disadvantages of each system, as well as to improve the quality of maternal and child care. The English version of this paper is available at: http://www.insp.mx/salud/index.html
\end{abstract}

Key words: home child birth/complications; natural child birth/complications; hospital care/complications; midwives, practical; Mexico

Agradecemos al Consejo N acional de Ciencia y Tecnología el apoyo para realizar este estudio (convenio p219C C 0 L880947).

(1) Departamento de Ecología Humana, Centro de Investigación y de Estudios Avanzados. Instituto Politécnico N acional, Unidad Mérida, Mérida,Yucatán, México.

Fecha de recibido: 8 de enero de 2001 - Fecha de aceptado: 19 de octubre de 2001

Solicitud de sobretiros: MISS Rosa María Méndez G. Departamento de Ecología Humana, Centro de Investigación y de Estudios Avanzados, Instituto Politécnico N acional, Unidad Mérida. Km 6 Antigua Carretera a Progreso, 97310 Mérida,Yucatán.

Correo electrónico:rmmg@mda.cinvestav.mx 
E n las últimas tres décadas, el modelo médico de atención del parto por vía vaginal, sin complicaciones, ha sido objeto de creciente escrutinio, ${ }^{1-3}$ debido a su tratamiento hospitalario y a la utilización rutinaria e innecesaria de tecnologías médicas que, además de ser incómodas para la mujer, pueden llegar a alterar su salud o la del recién nacido. Tal es el caso de la posición de litotomía que se utiliza por comodidad del médico, no obstante que favorece la aparición de desgarros perineales, además de tener efectos negativos sobre la ventilación pulmonar y la presión sanguínea de la madre, ${ }^{4,5}$ el rasurado del vello púbico que puede incrementar el riesgo de infección al producirse pequeñas laceraciones; ${ }^{6}$ la aplicación de enema, a pesar de que la expulsión de materia fecal durante el parto no aumenta la tasa de infecciones y de que no las previene; ${ }^{7}$ la administración de solución glucosada por vía parenteral, que puede reducir la tolerancia al dolor; ${ }^{8}$ la inducción y conducción química del parto que puede ocasionar hiperestimulación con alteraciones fetales por anoxia, hemorragia intracraneal, espasmos uterinos con posible separación prematura de la placenta, laceraciones del canal del parto y ruptura uterina, ${ }^{9-11}$ la episiotomía, aunque no previene desgarros, no es más fácil de reparar que éstos, ${ }^{12}$ no mejora la función de los músculos perineales ${ }^{13-15} \mathrm{y}$, además, puede producir laceraciones severas, dispareunia y pérdida sanguínea. ${ }^{16,17}$

Como resultado, en diversos países industrializados se han desarrollado modelos alternativos, en los que el parto es considerado un evento natural y es atendido, con nula o mínima utilización de tecnología médica, en hospitales, centros especiales o en el domicilio de la mujer por parteras-enfermeras o parteras profesionales, egresadas de escuelas de partería. ${ }^{18-20}$

Otra es la situación en países donde la medicina académica coexiste con una todavía fuerte medicina tradicional. En esta última, el parto es concebido como evento natural y su atención es competencia de las parteras tradicionales, quienes hacen uso de conocimientos y recursos comunitarios y pueden haber recibido cursos de capacitación, en cuyo caso recurren también a prácticas de la medicina académica.

En estos países, la atención se ha centrado, fundamentalmente, en la capacitación de las parteras tradicionales y su incorporación a los sistemas de salud para disminuir la mortalidad materna y neonatal. Esto se refleja en la literatura médica publicada de 1975 a la fecha, de la cual 63\% aborda estos temas. En menor proporción, se destaca la importancia de las parteras como agentes tradicionales de salud (23\%) y se describen las concepciones y prácticas tradicionales (14\%). De estos últimos, sólo dos evalúan dichas prácticas. ${ }^{21,22}$
Uno de ellos, ${ }^{22}$ realizado en el estado de Morelos, destaca la necesidad de diseñar programas de capacitación, a partir de la evaluación de las ventajas y desventajas de la atención brindada por las parteras tradicionales, considerando tanto la lógica del sistema cultural de la comunidad a la que pertenecen, y de la cual se derivan sus conocimientos y prácticas, así como los parámetros de la medicina académica.

La importancia de evaluar la calidad de la atención del parto por vía vaginal, ofrecida por el sistema hospitalario y por el sistema tradicional en México, queda destacada al considerar que $25.3 \%$ de la población nacional vive en localidades rurales ${ }^{23}$ y en éstas, $44.5 \%$ de los partos es atendido por parteras tradicionales. ${ }^{24}$ En otras palabras, la cobertura de la atención tradicional del parto es, aproximadamente, la misma que la hospitalaria para la población de zonas rurales depauperadas, en su mayoría, indígena. A ello se suma $23.7 \%$ de mujeres atendidas por parteras en localidades de 2500 a menos de 20000 habitantes. ${ }^{24}$

En Yucatán se ha estimado que 50\% de los partos es asistido por parteras. ${ }^{25}$ Los estudios sobre la atención tradicional del embarazo y del parto en el estado son de carácter antropológico médico. . $^{3,26-28}$ En ellos, se destaca su importancia dentro del grupo de agentes tradicionales de la salud y se describen y valoran positivamente las prácticas y procedimientos de atención. Contrastando con estos estudios, existe una percepción generalizada en el ámbito médico que atribuye a las parteras tradicionales la responsabilidad de la mortalidad materna y perinatal en el estado, por la mala atención que brindan. ${ }^{28}$ Sin embargo, no existe ningún estudio que evalúe las ventajas y desventajas de la atención del parto por vía vaginal otorgada por el sistema hospitalario y por el sistema tradicional en Yucatán.

Si bien, en teoría, no deben ocurrir complicaciones en la atención del parto por vía vaginal, es un hecho que se presentan. Por ello y ante la carencia de estudios que aporten elementos para mejorar su atención, se hizo una investigación cuyo propósito fue comparar la efectividad de dicha atención en los sistemas hospitalario y tradicional en Yucatán, partiendo de la hipótesis de que las diferencias en los procedimientos y recursos utilizados en cada sistema podrían ocasionar complicaciones maternas y neonatales diferentes.

\section{Material y métodos}

El estudio, realizado en 1989-1990, en el estado de Yucatán, México, fue de tipo transversal y comparativo. Se evaluó la atención del parto y del recién nacido brindada por un hospital de cada una de las instituciones 
más importantes del sistema nacional de salud, Secretaría de Salud (SSA), Instituto Mexicano del Seguro Social (IMSS) e Instituto de Seguridad y Servicios Sociales de los Trabajadores del Estado (ISSSTE), ubicados en la ciudad de Mérida, y por 21 parteras de los municipios de Valladolid, Temozón, Ticul y Halachó. Para su ejecución se obtuvo el consentimiento de las personas que atendieron el parto y de las mujeres estudiadas.

Se calculó una muestra de 216 mujeres, considerando la población femenina en el estado, el número de mujeres en edad reproductiva, la proporción de éstas que tuvo un parto y un intervalo de confianza de $95 \%$. Con base en la estimación de partos atendidos por parteras tradicionales $(50 \%)$, la muestra se dividió en igual proporción para cada uno de los sistemas estudiados. Las 108 mujeres estudiadas en el sistema hospitalario se distribuyeron de acuerdo con la cobertura de cada una de las tres instituciones oficiales de salud en el estado. En el tradicional no se pudo establecer la proporción de casos a estudiar por municipio, debido a que no existe información sobre su cobertura a ese nivel. Las mujeres estudiadas fueron seleccionadas, durante un periodo de seis meses, por muestreo sistemático entre aquellas que se presentaron para recibir atención del parto en cada sistema y sin complicaciones del embarazo. Se les observó desde el ingreso a la sala de labor en el hospital o desde que la partera acudió a su domicilio, hasta el posparto inmediato.

Se aplicaron dos instrumentos que consideraron prácticas comunes y específicas de los dos sistemas. El primero consistió en una guía de entrevista y de observación del proceso de atención del parto, dividida en dos partes. Una, enfocada a captar información demográfica, socioeconómica y ginecobstétrica y otra a registrar, mediante observaciones directas, las acciones y recursos utilizados durante la atención del parto y del recién nacido. El instrumento se aplicó en la sala de labor o cuando la partera había llegado al domicilio, estando ya la mujer en trabajo de parto. A los 15 días posparto, se visitó a las mujeres en su casa para identificar complicaciones maternas y neonatales que pudieran haberse derivado de la atención, utilizando una guía de entrevista que las interrogaba sobre signos y síntomas asociados con aquéllas. Las complicaciones maternas estudiadas en ambos sistemas fueron desgarros perineales, infección en desgarros, sangrado abundante durante el posparto, infección puerperal y muerte. Además, en el sistema hospitalario se incluyeron infección y dehicencia de la episiotomía, y flebitis por suero. En el caso de las complicaciones neonatales, se consideraron onfalitis, conjuntivitis neona- tal, neumonía por broncoaspiración, traumatismos obstétricos y muerte.

La aplicación de los instrumentos estuvo a cargo de tres pasantes de medicina y seis mujeres de los municipios. En un principio, se planeó que los primeros realizaran todas las observaciones. Sin embargo, durante las entrevistas de selección, manifestaron que las parteras "carecían de los conocimientos y preparación adecuados". Considerando que esta actitud podía llevarlos a introducir sesgos en la información, se decidió que levantaran sólo la información del sistema hospitalario y que mujeres de los municipios hicieran lo propio en el sistema tradicional.

Estas mujeres fueron seleccionadas de una lista proporcionada por las parteras y cumplieron con los criterios de ser bilingües maya-español, alfabetas, tener una buena relación con las mujeres y las parteras y ser respetuosas del trabajo de estas últimas.

Los nueve observadores fueron capacitados en métodos y técnicas de levantamiento de información, incluyendo el significado de cada uno de los ítems y la manera de formular y registrar las observaciones directas y las preguntas y las respuestas. Después de capacitados, se realizó una prueba piloto de los instrumentos en cada uno de los dos sistemas, en la que participaron con la autora principal. No hubo diferencias entre la información captada por esta última y el personal de campo, ni fue necesario hacer cambios en el contenido de los ítems.

La información fue procesada con el SPSS versión 6.0. Se utilizó la prueba de ji cuadrada para comparar los procedimientos comunes y el número de complicaciones maternas y neonatales entre sistemas, agrupando, por una parte, la información de los tres hospitales y de las parteras de los cuatro municipios, por otra.

\section{Resultados}

El número de casos con información completa se redujo a 205, de los cuales 101 corresponden al sistema hospitalario y 104 al tradicional. El cuadro I presenta información general de las mujeres. Las características socioeconómicas, incluyendo lugar de procedencia, fueron compatibles con el sistema de atención al que recurrieron. Así, la mayoría de las mujeres atendidas en los hospitales era de origen urbano y suburbano, tenía mejor nivel de escolaridad, mejores ingresos, poco más de la cuarta parte contaba con un empleo estable y tenía menos hijos. En cambio, en el sistema tradicional la mayoría era de origen rural, analfabeta o con primaria incompleta, y con ingresos familiares menores a un salario mínimo. 
El cuadro II presenta las acciones realizadas y los recursos utilizados para la atención del parto en ambos sistemas. El hospitalario se caracterizó por la aplicación de interrogatorio general y exploración física hechos en el servicio de admisión. Aunque con variaciones, se efectuaron exámenes de laboratorio. Posteriormente, las mujeres fueron trasladadas a la sala de labor, donde se les proporcionó una bata del hospital y permanecieron acostadas en una cama; se les rasuró el vello púbico; se les aplicó solución por vía parenteral, enema evacuante, anestesia epidural y oxitócicos. De ahí, se les llevó a la sala de expulsión, donde, en una mesa de puntos, se les colocó en posición de litotomía haciéndoseles antisepsia de la región perineal, episiotomía y, en el posparto, revisión de la placenta. El parto fue atendido por médicos internos de pregrado, generales y ginecobstetras. Al comparar entre hospitales, se encontró que en el ISSSTE el número de partos atendido por los últimos $(68 \%)$ fue significativamente mayor $\left(\chi^{2}=15.17, p=0.0005\right)$ que en el de la SSA $(25.6 \%)$ y en el del IMSS (24.3\%).

\begin{tabular}{|c|c|c|c|}
\hline \multirow{2}{*}{\multicolumn{4}{|c|}{ Cuadro I }} \\
\hline & & & \\
\hline DE LAS MUJER & OOR SISTEMA & DE ATENCI & \\
\hline YuCAT & Méxıco, 19 & $39-1990$ & \\
\hline & $\begin{array}{c}\text { Hospitalario } \\
n=101\end{array}$ & $\begin{array}{r}\text { Tradicional } \\
n=104\end{array}$ & $\begin{array}{c}\text { Total } \\
\mathrm{n}=205\end{array}$ \\
\hline Edad $(X \pm D E)$ & $24.7 \pm 5.2$ & $25.3 \pm 5.0$ & $24.9 \pm 5.1$ \\
\hline Paridad (\%) & & & \\
\hline Primípara & 29.7 & 9.6 & 19.7 \\
\hline Multípara & 70.3 & 90.4 & 80.3 \\
\hline Procedencia (\%) & & & \\
\hline Urbana/suburbana & 79.2 & 28.8 & 54.0 \\
\hline Rural & 20.8 & 71.2 & 46.0 \\
\hline Escolaridad (\%) & & & \\
\hline Analfabeta & 12.9 & 33.7 & 23.3 \\
\hline Primaria incompleta & 24.8 & 33.7 & 29.2 \\
\hline Primaria completa & 25.7 & 16.3 & 21.0 \\
\hline Secundaria y más & 36.6 & 16.3 & 26.5 \\
\hline 0 cupación (\%) & & & \\
\hline Hogar & 73.2 & 91.3 & 82.2 \\
\hline Empleo remunerado & 26.8 & 8.7 & 37.8 \\
\hline Ingresos familiares (\%) & & & \\
\hline$<1$ salario mínimo & 1.0 & 57.7 & 29.3 \\
\hline$>1$ salario mínimo & 99.0 & 42.3 & 70.7 \\
\hline Servicio sanitario (\%) & 66.3 & 28.8 & 47.5 \\
\hline
\end{tabular}

\section{Cuadro II \\ ACCIONES REALIZADAS Y RECURSOS UTILIZADOS DURANTE LA ATENCIÓN DEL PARTO POR SISTEMA de atención.Yucatán, Méxıco, 1989-1990}

$\begin{array}{ccc} & \text { Hospitalario } & \text { Tradiciona } \\ \% & \% \\ \text { Mala higiene de vivienda } & \text { na } & 26.0\end{array}$

\begin{tabular}{lll}
$\begin{array}{l}\text { Exámenes de laboratorio } \\
\text { Biometría hemática }\end{array}$ & 94.1 & na \\
\hline Q uímica sanguínea & 84.2 & na \\
\hline Examen general de orina & 74.3 & na
\end{tabular}

\begin{tabular}{lll} 
Soluciones parenterales & 100.0 & na \\
\hline Masajes abdominales & na & 14.4 \\
\hline Anestesia epidural & 15.8 & na \\
\hline Oxitócicos (inducción y conducción) & 47.5 & na \\
Hamaca sucia & na & 7.7 \\
\hline Equipo de parto disponible & na & 68.3 \\
\hline Antisepsia de región perineal & $99.0^{*}$ & 73.1 \\
\hline Ruptura de bolsa amniótica & 0 & 2.9 \\
\hline Episiotomía & 77.2 & na \\
\hline Revisión de placenta & $99.0^{\ddagger}$ & 76.9
\end{tabular}

na: no se aplica

$*\left(\chi^{2}=28.37, p=0.000001\right)$

$\ddagger\left(\chi^{2}=23.34, p=0.000001\right)$

En el sistema tradicional, los partos fueron atendidos en las viviendas de las mujeres. Las parteras suelen recomendarles tener disponibles dos hamacas limpias, una para la atención del parto y la otra, para que madre e hijo descansen. Es costumbre que la partera arribe una vez que la mujer presente los dolores de parto. Aunque se observaron diferencias, durante el trabajo de parto, la mujer pudo caminar y se hicieron masajes abdominales o "sobadas" para estimular las contracciones uterinas y acomodar el tipté.* En el momento del parto, la mujer se sentó en una hamaca, extendió sus brazos y se agarró con fuerza de los extremos de ésta; el esposo u otro familiar cercano se colocó detrás, poniéndole los brazos debajo de las axilas para que se apoyara con seguridad. Se realizaron antisepsia de la región perineal y ruptura de la bolsa amniótica, prácticas aprendidas por las parteras que

* De acuerdo con la tradición maya de Yucatán, el tipté o cirro es el órgano rector del cuerpo humano que marca su centro y lo divide en cuatro áreas, en forma similar al cosmos. Se ubica debajo del ombligo. Cuando es desplazado por otros órganos, como sucede durante el embarazo, se manifiestan alteraciones en los individuos y se requieren dichas "sobadas". 
han tomado cursos de capacitación. En el posparto revisaron la placenta. La edad promedio de las parteras fue de $61.3 \pm 4.7$ años y $47.6 \%$ había recibido cursos de capacitación. El número de parteras capacitadas de Ticul (77.8\%) fue significativamente mayor (Fisher $p=0.030$ ) que el de las de los otros tres municipios juntos $(25 \%)$.

Se encontraron diferencias significativas en dos de los tres procedimientos comunes a ambos sistemas: antisepsia de la región perineal y revisión de la placenta, los cuales fueron practicados con mayor frecuencia en el sistema hospitalario.

En el cuadro III se presentan las acciones realizadas y los recursos para la atención del neonato en ambos sistemas. En el hospitalario se emplearon aspiradores o perillas para extraer las secreciones nasofaríngeas; tijeras desinfectadas y cintas umbilicales para amarrar el cordón y, en todos los casos, se contó con báscula y gotas oftálmicas. En el tradicional, las secreciones fueron extraídas por la partera en forma manual, envolviendo sus dedos en algodón o mediante perilla; se utilizaron cintas de trapo, hilos de hamaca o cintas umbilicales, cortándose el cordón con navajas de rasurar o tijeras. No todas las parteras contaban con báscula y gotas oftálmicas. Después del parto, las parteras bañaron a los recién nacidos.

Como en el caso de la atención del parto, se encontraron diferencias significativas en dos de los tres procedimientos comunes a los sistemas: aplicación de gotas oftálmicas y registro del peso al nacer, los cuales fueron realizados con mayor frecuencia en el sistema hospitalario.

Una comparación al interior de cada uno de los sistemas reveló diferencias significativas en la atención entre hospitales, y entre parteras. En el hospitalario, sólo se encontró diferencia en la exploración física del recién nacido. En el sistema tradicional se observaron diferencias en la antisepsia de la región perineal, la higiene de la vivienda, la disponibilidad del equipo de parto, la revisión de la placenta y la práctica de masajes abdominales o "sobadas", los que, de acuerdo con nuestras observaciones, más que estimular las contracciones uterinas proporcionan alivio al dolor durante éstas. Asimismo, se encontraron diferencias en la aplicación de gotas oftálmicas y en la obtención del peso del neonato. ${ }^{29}$

Como hemos señalado, aunque no deberían ocurrir complicaciones en la atención del parto por vía vaginal, éstas se presentan. El cuadro IV muestra que las complicaciones maternas fueron más frecuentes que las neonatales. Las primeras afectaron a $33.4 \%$ de las mujeres pero no se presentó ningún caso de cesárea. Las segundas a $10.6 \%$ de los recién nacidos.

\begin{tabular}{|c|c|c|}
\hline $\begin{array}{l}\text { Cuadro II } \\
\text { ACCIONES REALIZADAS Y REC } \\
\text { DURANTE LA ATENCIÓN DEL RECI } \\
\text { DE ATENCIÓN.YUCATÁN, M }\end{array}$ & $\begin{array}{l}\text { RSOS UTILIZ } \\
\text { NACIDO PO } \\
\text { ICO, 1989- }\end{array}$ & $\begin{array}{l}\text { ADOS } \\
\text { SISTEMA } \\
990\end{array}$ \\
\hline & $\begin{array}{c}\text { Hospitalario } \\
\%\end{array}$ & $\begin{array}{c}\text { Tradicional } \\
\%\end{array}$ \\
\hline Aspiración de secreciones nasofaríngeas & 100.0 & 100.0 \\
\hline Exploración física & 88.0 & na \\
\hline A plicación de gotas oftálmicas & $100.0^{*}$ & 56.7 \\
\hline Registro de peso al nacer & $100.0^{\ddagger}$ & 51.5 \\
\hline $\begin{array}{l}\text { Corte del cordón umbilical con instrumer } \\
\text { desinfectado }\end{array}$ & na & 97.1 \\
\hline $\begin{array}{l}\text { A marre del cordón umbilical con } \\
\text { trapo/hilo limpio o cinta umbilical }\end{array}$ & na & 99.0 \\
\hline $\begin{array}{l}\text { na: no se aplica } \\
*\left(\chi^{2}=55.99, p=0.000001\right) \\
\ddagger\left(\chi^{2}=70.21, p=0.000001\right)\end{array}$ & & \\
\hline
\end{tabular}

\section{Cuadro IV \\ Complicaciones maternas y neonatales por sistema de atención.Yucatán, México, 1989-1990}

$$
\begin{array}{cc}
\text { Hospitalario } & \text { Tradicional } \\
\% & \%
\end{array}
$$

Complicaciones maternas

\begin{tabular}{llc} 
Desgarros perineales & 9.9 & 5.8 \\
\hline Infección en desgarros perineales & 1.0 & 0 \\
\hline Sangrado abundante en el posparto & 1.9 & 4.8 \\
\hline Infección o dehicencia de la episiorrafia & 5.0 & na \\
\hline Flebitis por suero & 1.9 & na \\
\hline Infección puerperal & 3.0 & 0 \\
\hline Muerte & 0 & 0 \\
\hline Total & $22.8^{*}$ & 10.6
\end{tabular}

Complicaciones neonatales

\begin{tabular}{lll} 
O nfalitis & 1.0 & 1.9 \\
\hline Conjuntivitis neonatal & $0^{\ddagger}$ & 6.7 \\
\hline Traumatismos obstétricos & 0 & 1.0 \\
\hline N eumonía por broncoaspiración & 0 & 0 \\
\hline Muerte & 0 & 0 \\
\hline Total & $1.0^{5}$ & 9.6
\end{tabular}

na: no se aplica

$*\left(\chi^{2}=4.66, p=0.03\right)$

₹ (Fisher $p=0.01$ )

$\S\left(\chi^{2}=5.90, p=0.01\right)$ 
En conjunto, en el sistema hospitalario se presentó un número significativamente mayor de complicaciones maternas que en el sistema tradicional y en éste se presentaron más complicaciones neonatales que en el primero. Al comparar cada una de las complicaciones, no se encontraron diferencias significativas en el caso de las maternas pero sí en el de las neonatales. La conjuntivitis neonatal fue más frecuente en el sistema tradicional que en el hospitalario.

Finalmente, se comparó el número total de complicaciones entre sistemas, sumando el número de complicaciones maternas y neonatales. En el hospitalario se presentaron complicaciones en $23.8 \%$ de los casos y en el tradicional en $20.2 \%$. La diferencia no fue estadísticamente significativa $\left(\chi^{2}=0.20, p=0.65\right)$.

\section{Discusión}

Los resultados muestran la existencia de complicaciones maternas y neonatales, aun cuando se trata de partos por vía vaginal. Las complicaciones observadas se relacionan con los procedimientos y recursos utilizados para la atención del parto y del recién nacido. Así, se encontró que las complicaciones maternas fueron más frecuentes que las neonatales, aunque su distribución en cada sistema fue diferente. Mientras que las primeras fueron más frecuentes en el sistema hospitalario, en el tradicional se presentaron con mayor frecuencia las complicaciones neonatales.

La mayor frecuencia de complicaciones maternas en el sistema hospitalario puede atribuirse al manejo inadecuado y a la utilización de tecnologías médicas innecesarias en la atención del parto por vía vaginal. Los partos fueron atendidos por personal médico con diferente grado de especialización, sin que hubiera criterios que definieran el perfil o características de adiestramiento necesarios para su atención. El empleo indiscriminado de tecnologías puede aumentar el riesgo de complicaciones maternas y neonatales y, además, contribuir al encarecimiento del servicio. La complicación más frecuente fue el desgarro perineal, seguida por la infección o dehicencia de la episiotomía. Aunque la posición de litotomía puede incrementar el riesgo de desgarros perineales, la causa principal de éstos es el manejo inadecuado del periné durante la fase expulsiva del parto. La infección y la dehicencia se añaden a los riesgos de la episiotomía y pudieron ser consecuencia del empleo de materiales o instrumentos contaminados. Aunque la infección pudo también haber sido causada por negligencia de las mujeres. En un estudio realizado en un hospital de la Ciudad de México, la infección de la episorrafia ocupó el segundo lugar entre las infecciones nosocomiales posparto. ${ }^{30}$

En el sistema tradicional, la complicación materna más frecuente fue también el desgarro perineal, la cual, como señalamos, es consecuencia de un manejo inadecuado del parto. La mayor frecuencia de complicaciones neonatales en el sistema tradicional puede atribuirse a la falta de recursos o a un manejo inadecuado. Específicamente, la conjuntivitis neonatal fue la complicación más frecuente y sólo se presentó en este sistema. Es interesante señalar que todos los casos se presentaron en Ticul, a pesar de que la mayoría de las parteras había recibido cursos de capacitación y de que aplicaron gotas oftálmicas a $88.1 \%$ de los neonatos.

En términos de la evaluación de servicios de salud, estos resultados sugieren que la atención del parto por vía vaginal y del recién nacido en ambos sistemas es comparable en cuanto a calidad. Es decir, en los dos existe el riesgo de que se presenten complicaciones maternas y neonatales. En el caso de las primeras, se observó que en ambos se presentaron complicaciones severas que pueden poner en riesgo la vida de la madre (sangrado abundante en el posparto e infección puerperal). Sin embargo, en el sistema hospitalario se observó un mayor número de complicaciones menos severas que, si bien no comprometen la vida de la mujer, sí afectan su salud, estando algunas de ellas ligadas a la tecnología médica (infección o dehicencia de episiotomía y flebitis por suero). En cuanto a las complicaciones neonatales, se observó una mayor frecuencia en el sistema tradicional, aunque la mayoría de los casos se refiere a una complicación que no compromete la vida del recién nacido (conjuntivitis).

No obstante que, por tratarse de partos por vía vaginal, el número de complicaciones fue relativamente pequeño, éstas pueden atribuirse a deficiencias en los procedimientos y recursos utilizados y, por tanto, pudieron ser prevenidas. En el sistema hospitalario, las deficiencias pueden relacionarse con la falta de entrenamiento adecuado del personal y al uso indiscriminado de recursos y tecnologías médicas, y en el sistema tradicional a la carencia de recursos mínimos indispensables, a la falta de capacitación o de calidad de la misma.

Es interesante hacer notar que en el sistema hospitalario el conjunto de procedimientos y recursos utilizados para la atención del parto y del recién nacido fue relativamente homogéneo, mientras que en el sistema tradicional se observó una variación relativamente grande. Esto puede deberse a la lógica que subyace a cada sistema, la cual se deriva de con- 
cepciones diferentes sobre el ser humano y la naturaleza. ${ }^{22}$ En el caso del primero, se trata de un saber institucionalizado, cuya transmisión involucra el aprendizaje escolarizado. En el segundo, se trata de un saber subordinado cuyo aprendizaje involucra la transmisión oral y el aprendizaje empírico. Estas diferencias son rara vez consideradas en el diseño de programas de capacitación para personal de salud y parteras, y del currículo de la carrera de medicina.

Son escasos los estudios que evalúan los recursos y procedimientos utilizados por parteras tradicionales y sus ventajas y desventajas. Los pocos que lo han hecho han evaluado positivamente los masajes abdominales ${ }^{22}$ y la posición de cuclillas para el parto. ${ }^{21,31}$

Desde luego, es necesario considerar las limitaciones de este estudio. Primero, fue pequeño debido a que se enfocó a evaluar la atención del parto por vía vaginal, el número de eventos observados, es decir, las complicaciones maternas y neonatales. Segundo, se realizó antes de que se estableciera la Norma Oficial Mexicana para la atención de la mujer durante el embarazo, parto y puerperio y del recién nacido ${ }^{32}$ y de las recomendaciones de la Organización Mundial de la Salud (OMS), ${ }^{33}$ que suprimen procedimientos médicos rutinarios e innecesarios.

Sin embargo, los resultados encontrados son congruentes con los estudios que evalúan la tecnología médica y las prácticas de parteras tradicionales y sugieren la necesidad de llevar a cabo estudios que, como el presente, evalúen los recursos y procedimientos que cada sistema utiliza para atender el parto por vía vaginal y al recién nacido. Independientemente de que provengan de los saberes y prácticas tradicionales o de la medicina académica, es necesario identificar aquellos que por sí mismos o por su utilización incorrecta o indiscriminada representan un riesgo para la salud materno-infantil, así como aquellos que aseguran una atención con calidad y calidez, ${ }^{32}$ además de adecuada al contexto en el cual viven las mujeres. De esta manera, este tipo de estudios contribuiría a una evaluación objetiva de los sistemas hospitalario y tradicional, la cual, aunada a estudios de carácter antropológico médico, permitiría valorar el importante papel que juegan las parteras en la salud materno-infantil, así como al diseño y evaluación de programas de capacitación, que consideren sus necesidades y prácticas, permitan su participación activa y den seguimiento a las acciones de supervisión y provisión de recursos. Finalmente, estudios de este tipo también podrían contribuir a evaluar el impacto de la Norma Oficial y de las recomendaciones de la OMS sobre la práctica médica y la propia capacitación de las parteras empíricas.

\section{Agradecimientos}

Agradecemos al personal de los hospitales, a las parteras, a los pasantes de medicina, a las mujeres que levantaron la información y a las participantes.

\section{Referencias}

1. Chalmers I. British debate on obstetric practice. Pediatrics 1976;58: 308-311.

2. Torres A, Reich M. The shift from home to institutional childbirth: A comparative study of the U nited Kingdom and the $\mathrm{N}$ etherlands. Int J H ealth Serv 1989;19:405-414.

3. Jordan B. Birth in four cultures. A crosscultural investigation of childbirth in Yucatan, Holland, Sweden and the U nited States. $4^{a}$ edición. C hicago:W aveland, 1993.

4. Caldeyro BR. Effect of position changes on the intensity and frequency of uterine contractions during labor. Am J 0 bstet Gynecol 1970;80: 284-290.

5. Hugo M. A look of maternal position during labor. J N urse Midwifery 1977;12:22-26.

6. Seropian R, Reynolds B.W ound infections after preoperative depilatory versus razor preparation. Am J Surg 1971;121:251-258.

7. Romney M, Gordon $\mathrm{H}$. Is your enema really necessary? $\mathrm{Br}$ Med J 1981;282:1269-1274.

8. Morley G,Arshag D M, Allen L, Morley J. Mechanism of pain in diabetic peripheral neuropathy: Effect of glucose on pain perception in human.Am J Med 1984;47:79-82.

9. Fields H. Induction of labor: Methods, hazards, complications and contraindications. Hosp Top 1968;46:63-66.

10. Baird D. Induction of labour. Br Med J 1976;1:896-916.

11. MacN ay MB, McLawaine GM, Howie PW, MacN aughton MC. Perinatal deaths:Analysis by clinical cause to asses the value of induction of labour. Br Med J 1977;301:247-350.

12.Tacker SB, Banta D. Benefits and risks of episiotomy: $A n$ interpretative review of the English language literature, 1960-1980. 0 bstet G ynecol Surv 1983;38:322-338.

13. Gass MS, D unn CD, Stys SJ. Effect of episiotomy on the frequency of vaginal outlet lacerations. J Reprod Med 1986;31:240-244.

14. Goer H. O bstetric myths versus research realities: A guide to the medical literature.W estport:Bergin and Garvey,1995.

15. Klein MC, G authier RJ, Robbins JM, Kaczorowski J, Jorgensen SH, Franco ED et al. Relationship of episiotomy to perineal trauma and morbidity, sexual dysfunction, and pelvic floor relaxation. Am J 0 bstet Gynecol 1994;171:591-598.

16. Borgatta L, Piening SL, C ohen W R.Association of episiotomy and delivery position with deep perineal laceration during spontaneous delivery in nulliparous women. Am J O bstet Gynecol 1989;160:294-297.

17. LaVoie A. Episiotomy may be overused. Am J O bstet Gynecol 1997;176:411-414.

18. Kerssens J. Patient satisfaction with home-birth care in The N etherlands. J Adv N urs 1994;20:344-350.

19.Tew M.The case against hospital deliveries:The statistical evidence. En: Kitzinger S, Davis J, ed. The place of birth. Londres: 0 xford University Press, 1978:55-65.

20. N ational C enter for Health Statistics. N ew study shows lower mortality rates for infants delivered by certified nurse midwives. Rockville: $\mathrm{N}$ ational Center for Health Statistics Press 0 ffice, 1998;301:436-551.

21. Ityavyar DA.A traditional midwife practice, Sokoto State, N igeria. Soc Sci Med 1984;18:497-501. 
22. Castañeda X, García C, Romero X, N úñez-U rquiza RM, González D, Langer A. Traditional birth attendants in Mexico: Advantages and inadequacies of care for normal deliveries. Soc Sci Med 1996;43:199-207.

23. Instituto N acional de Estadística, Geografía e Informática. XII Censo General de Población y Vivienda, 2000. Resultados Preliminares. Disponible en: http://www.inegi.gob.mx.

24. Secretaría de Salud. Subsecretaría de Servicios de Salud. Encuesta $N$ acional sobre Fecundidad y Salud. México, D.F.: SSA, 1989.

25. Menéndez E. Poder, estratificación y salud. México, D.F:: Ediciones de la Casa Chata, CIESAS, 1981.

26. Favier A. Parto y tradición. En: Memorias del Seminario sobre Capitalismo y Vida Rural en Yucatán. Mérida: U niversidad Autónoma de Yucatán, 1984:245-252.

27.G üemez MA. Estado actual de las prácticas médicas tradicionales en Pustunich, Yucatán. 1984. (Tesis de Licenciatura en Ciencias Antropológicas). Mérida, Yucatán, México: U niversidad Autónoma de Yucatán, 1984.

28. G uzmán MG. Concepciones de la salud y enfermedad en una comunidad campesina de Yucatán. 1992. (Tesis de Maestría en Antropología Antropología Social). México, D.F.: Escuela Nacional de Antropología e Historia, 1992.
29. Méndez RM. Evaluación comparativa de dos métodos de atención del parto y del recién nacido en Yucatán. Tesis de Maestría en Investigación de Servicios de Salud. México, D.F.: U niversidad N acional Autónoma de México, C ampus Iztacala, enero 2000.

30. Figueroa D R, O rtiz FJ,A rredondo JL. Infecciones nosocomiales de origen gineco-obstétrico en un hospital de atención perinatal. Salud Publica Mex 1994:36:10-16.

31. Romond JL, Baker IT. Squatting in childbirth. A new look at an old tradition. J 0 bstet Gynecol N eonatal N urs 1985;14:406-411.

32. Secretaría de Salud. N orma 0 ficial Mexicana N O M-007-SSA2-1993. Atención de la mujer durante el embarazo, parto y puerperio y del recién nacido. Criterios y procedimientos para la prestación del servicio. México, D.F.: Secretaría de Salud, Comité Consultivo $\mathrm{N}$ acional de N ormalización de Servicios de Salud, 1993.

33. W orld Health $\mathrm{O}$ rganization. C are in normal birth: Report of a technical working group. 1997.W HO/FRH/MSM/96.24. Disponible en: http:// www.who/int/reproductive-health/publications. 